\title{
Research on the Functions Fusion of Vehicle Information System
}

\author{
YinpengFei ${ }^{1,}$,, Li Juan ${ }^{2, b}$, \\ ${ }^{1}$ Computer Science and Technology, Beijing University of Technology, Beijing, 100124, China \\ ${ }^{2}$ Computer Technology, Beijing University of Technology, Beijing, 100124, China \\ aemail:yinpengfei1124@163.com, bemail:kuailelijuan1991@126.com
}

\begin{abstract}
Keywords: Vehicle Information System; Vehicle Network; Functions Fusion; Security Network Communication; Vehicle Data Simulation
\end{abstract}

\begin{abstract}
In view of the traditional vehicle information systems cannot satisfy the modern vehicle needs. Using the simulation software on the vehicle information system,based on the mutual cooperation of four computers, researching the functions fusion.Experiments show thatthe function of vehicle information system functions fusionare feasible, which can meet the needs of the modernization of the vehicle information system.It provides a reliable reference for the deployment of the application in the real environment.
\end{abstract}

\section{Introduction}

Vehicle has always been an important means of transport. With the development of information technology, the traditional single function of Vehicle System [1] cannot meet the needs of modern vehicles.In Vehicle Information System [2], having a very highly requirement on the function of information processing, which needs multi information collection and multi-function processing [3].

The modern information system is very complex, which needs a lot of information on the vehicle [4]. Including vehicle information, vehicle information, audio and video information, location and navigation information, vehicle maintenance information, statistics and decision information, vehicle information, vehicle information, vehicle information and auxiliary driving information [5]. Each kind of information collection will involve a lot of operating functions [6]. Therefore, a computer has been unable to meet the processing requirements of the vehicle information system, which needs to cooperate with each other to achieve.

Direct analysis of the functions fusion of vehicle information system will be very difficult, which needs a complicated environment [7]. Using simulation software [8] to replace real system environment,to product data generated during vehicle operation. The experiment based on the four computer, each computer belonging to different functions, including: machine operation control, information storage, safety network communication, public network communication[9]. Based on the mutual cooperation of these four computers, researching the functions fusion of vehicle information system.

\section{Design of the Functions Fusion of Vehicle Information System}

The simulation environment of vehicle information system is established by the following steps:

By researching the vehicle information system, the data generated during the operation of the vehicle are analyzed.Using the software to simulate the environment of the vehicle running, so that the multi computer can obtain the vehicle running data generated by the software, and it must ensure the consistency of data.

According to the vehicle information system function modules, each computer belong to different functional modules, to complete man-machine interface design and development.

By the analysis and design of the database, saving data from the vehicle running time and the data generated by communication between the computers.

Establishing a secure communication mechanism, whichwe can communicate with each other, cooperate with each other, to complete the monitoring, control, information storage and so on. 
In order to better describe the research plan on the functions fusion of the vehicle information system, the structure model of the vehicle information system is demonstrated by the topology structure diagram. The vehicle information system includes four computer hosts, each computer belonging to different functions. Including: machine operation control, information storage, safety network communication, public network communication. The topological structure of vehicle information is shown in Figure 1:

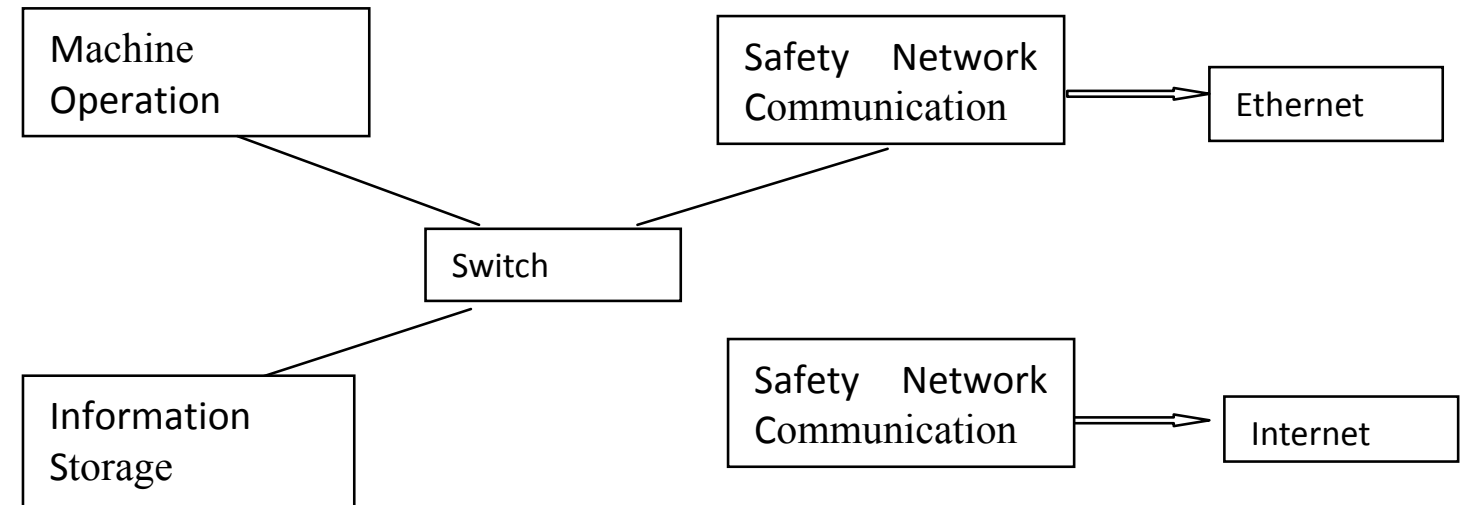

Fig.1. Topological structure of vehicle information system

A simple description of the function of each computer host:

Machine Operation Control:The main function is to realize the vehicle operation control, for the core components of the vehicle information system.

Information Storage: Realize the centralized storage and management of the relevant information of the vehicle.

Secure Network Communication: As a safety communication channel of the vehicle information system, it is the mainly communication by the vehicle information system local area network, which can be the connection between the vehicle or the vehicle and the remote command center.

Public Network Communication machine: Vehicle information system and the outside world of communication channels, mainly to achieve the connection with the outside world, and it is physical isolation with information network, the main function is to vehicle drivers to understand the vehicle external information.

\section{Realization of functions fusionof vehicle information system}

First, use the data synchronization program to simulate the vehicle running environment, which can continue to generate data for the vehicle running. These data conform to a certain vehicle operating rules, and then use the network technology to send data to alldifferent computers at the same time. When all of these computers receive data and send back to the determination of the message, it is considered that the data reached a synchronization, otherwise it is considered not to achieve synchronization, the re- transmission. The architecture of the software uses the master-slave structure, the structure diagram of the data synchronization program is given in Figure 2:

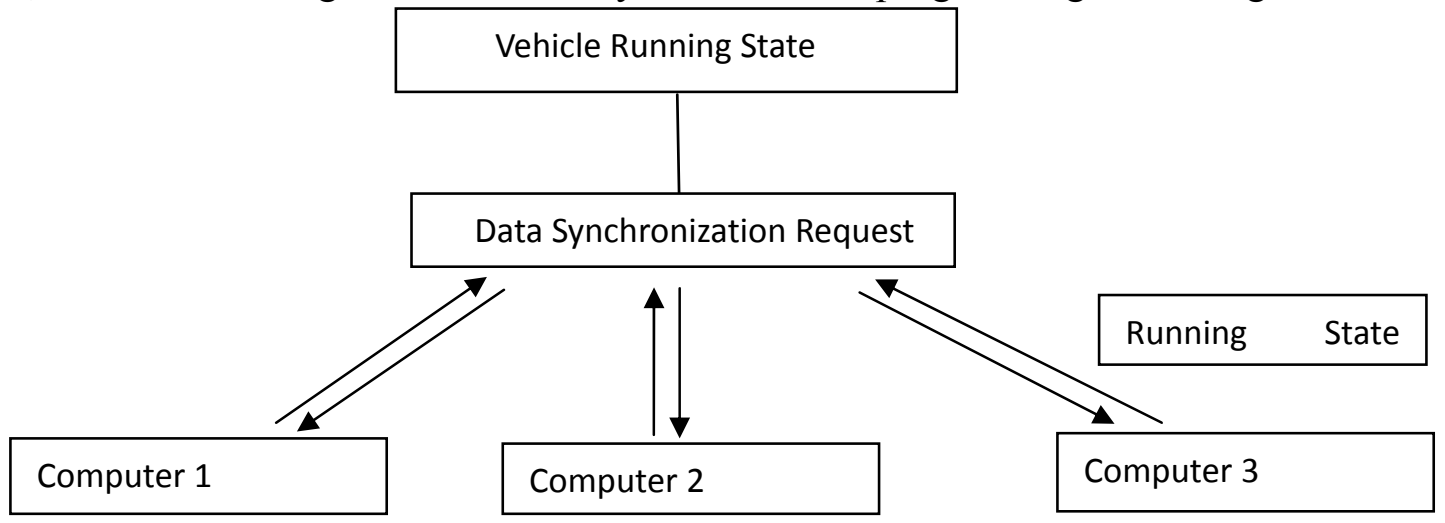

Fig.2.the structure diagram of the data synchronization program 
The interactive interface style of the system is combined with the real environment, and the LCD display style [9] is drawn with black background and blue lines. The vehicle state information is described by the dynamic line graph, which is by the technology of multi thread and double buffer. An interface effect diagram is given inFigure3:

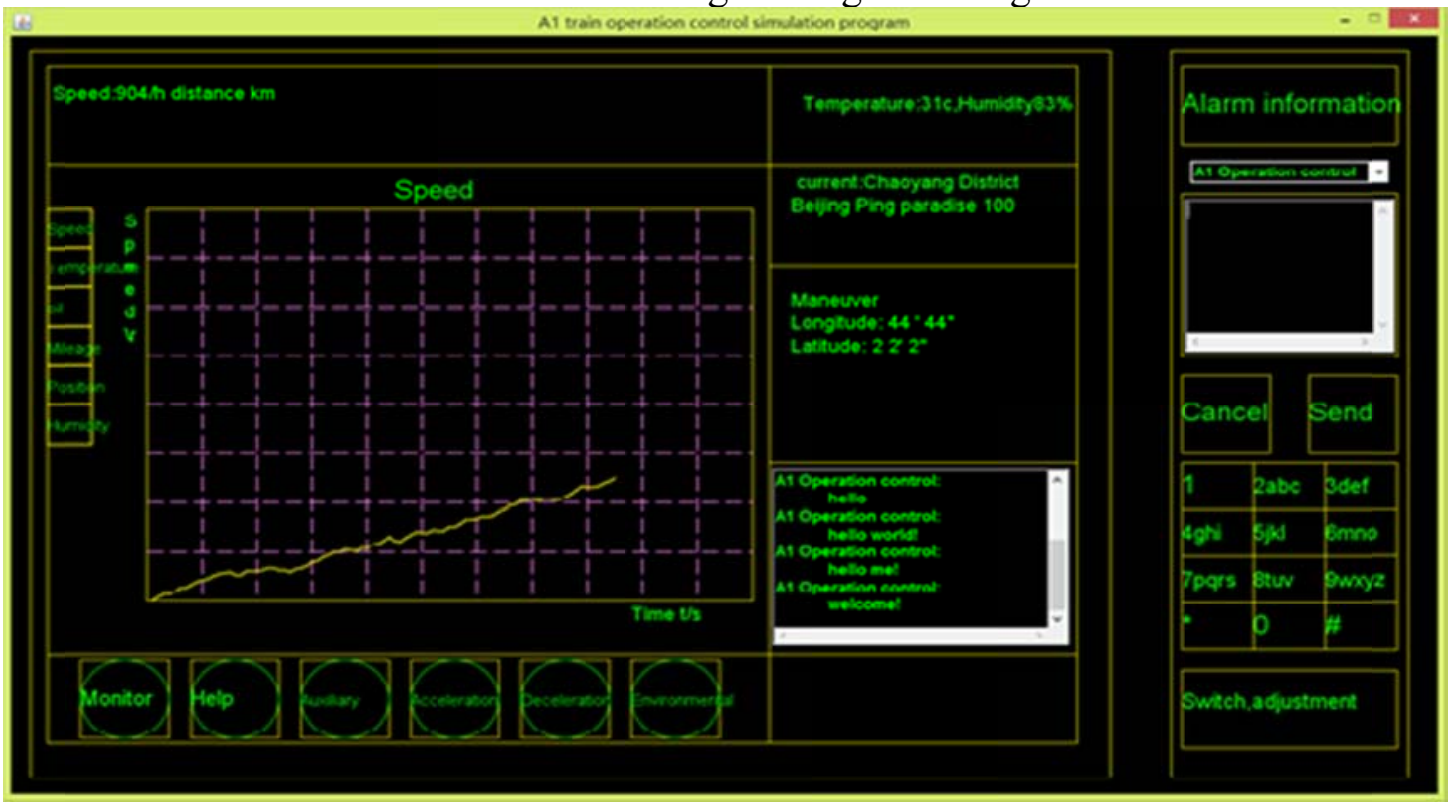

Fig.3 an interface effect diagram

The database design of the software is based on the data acquisition parameters of the vehicle information system, which has a high similarity, which can reflect the data information of the vehicle information system. We use MySQL database to store data.

About the network communication, combining the master-slave structure and the structure of P2P. A temporary service machine is derived from the program, and the rest of the machine access server to obtain data source and the corresponding operation.

When the server fails, then elected a new temporary server to act as the role of the main server. The structure can guarantee the network's fast and stable. Network topology diagram is given Figure 4:

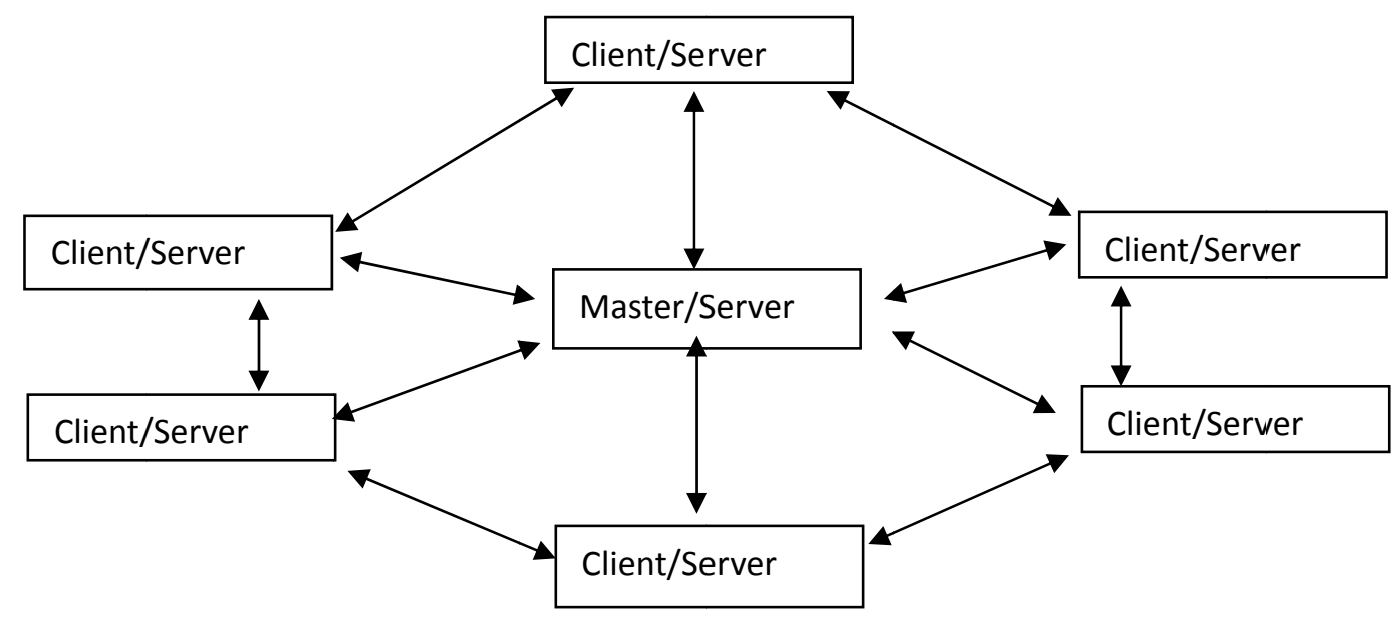

Fig.4.network topology diagram

The technical route of this project is to use Java technology to realize the program development of the vehicle information functions fusion, and complete the storage and query of information through the MySQL database. At the same time, the program has a good software system structure, which is in accordance with the software design pattern of MVC. 


\section{Conclusion}

In order to study the function of the vehicle information system, the project has taken a series of simulation program to carry on the experiment. The state of the vehicle running state and the realization of the vehicle information system are simulated by the Java related technology. The functions fusion of the vehicle information system is completed by four computers. Experimental results show that the functions fusion of the vehicle information system can be realized by multiple computers, and each computer belong to different functional modules which make a better use of vehicle information system data. It provides a reliable reference for the deployment of the application in the real environment.

\section{References}

[1] Xu Yang,Wilfred W. Recker. Modeling Dynamic Vehicle Navigation in a Self-Organizing, Peer-to-Peer, Distributed Traffic Information System[J].Journal of Intelligent Transportation Systems, 2006, Vol.10

[2] Han-Chun,Yeh,Su-YingChang, Yuan-ShengChu,Chien-WeiChen,Chi-Sheng

Shih.Vehicle Information Systems Integration Framework[J]. J. Inf. Sci. Eng., 2007, Vol.23

[3] Huang Zuowei, Zhou Ming, Zhang Ximei. Design and Realization of Embedded Vehicle Termi nal Based on GPS / GPRS. Computer Measurement \& control, Magn. China, vol. 17, no. 11, pp. 22 05-2208, 2009.

[4] Ran Li,Junfeng Wu,Haiying Wang. Design method of CAN BUS network communication structure for electric vehicle[A].Ulsan,Korea: IEEE,2010.

[5]Ronald Ecker, Verena Broy, Katja Hertzschuch, Andreas Butz . Visual cues supporting direct touch gesture interaction with in-vehicle information systemsRonald Ecker, Verena Broy, Katja Hertzschuch, Andreas Butz[J]. MobileHCI '09: Proceedings of the 11th International Conference on Human-Computer Interaction with Mobile Devices and Services September 2009.

[6]An extended keystroke level model (KLM) for predicting the visual demand of in-vehicle information systems[J]. CHI '07: Proceedings of the SIGCHI Conference on Human Factors in Computing Systems April 2007

[7]Yujia Cao, Frans van der Sluis, Mariët Theune, Rieks op den Akker, Anton Nijholt. Evaluating informative auditory and tactile cues for in-vehicle information systems [J]. AutomotiveUI '10: Proceedings of the 2nd International Conference on Automotive User Interfaces and Interactive Vehicular Applications November 2010

Michael Pettitt, Gary Burnett, Alan Stevens.

[8] Lars B, Frerdriksson. CAN for critical embedded automotive networks[J].IEEE Micro,2002,(04):28-35.doi:10.1109/MM.2002.1028473.

[9] Analog Device Inc. EE-256 using the ADSP-BF561 blackfin(R)processor as a TFT-LCD controller[OL].http://www,analog,com/en/index,html,2010.

[10]Chen I-An,Cheng Chang-Hsin,Jheng Hong-Yuan. An error-correction scheme with Reed-Solomon codec for CAN bus transmission[A].Chiang Ma,Thailand: IEEE,2011. 